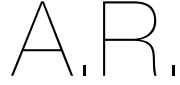

1 Escola Superior de Tecnologia da Saúde de Coimbra do Instituto Politécnico de Coimbra, Rua 5 de Outubro - São Martinho do Bispo, 3046-854 Coimbra Portugal

2 Departamento de Dietética e Nutrição, Escola Superior de Tecnologia da Saúde de Coimbra do Instituto Politécnico de Coimbra,

Rua 5 de Outubro - São Martinho do Bispo, 3046-854 Coimbra, Portugal

Endereço para correspondência: Ana Rita Fialho Rua Infante Dom Henrique, n. 273

3045-081 Coimbra, Portuga ana.rita fialho@hotmail.com

Histórico do artigo:

Recebido a 14 de maio de 2020 Aceite a 29 de junho de 2020

\title{
FUCOXANTINA: A SOLUÇÃO PARA A OBESIDADE?
}

\author{
FUCOXANTHIN: A SOLUTION TO OBESITY?
}

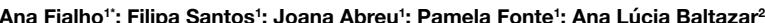

\section{RESUMO}

A fucoxantina é um dos carotenóides mais abundantes no ambiente marinho, podendo ser encontrada em microalgas e macroalgas. Atualmente, verifica-se um aumento de estudos com este composto, devido aos seus múltiplos benefícios para a saúde, nomeadamente o seu efeito anti-obesogénico.

O presente artigo de revisão narrativa pretende caracterizar os processos de metabolização e toxicidade, bem como os mecanismos de ação da fucoxantina no controlo da obesidade.

Esta revisão foi realizada na base de dados "PubMed" usando os descritores "fucoxantina", "efeito anti-inflamatório" e "obesidade". A partir da fonte mencionada, analisaram-se 44 artigos tendo sido selecionados 28, publicados entre 2015 e 2020.

Após a administração de fucoxantina, esta é rapidamente metabolizada em fucoxantinol. Parte deste metabolito é subsequentemente desidrogenado para amarouciaxantina A no fígado. Estudos demonstraram que a fucoxantina tem propriedades terapêuticas, tendo um efeito notável na obesidade, através da estimulação da expressão de UCP1. Esta proteína reduz o tecido adiposo branco e inibe a acumulação intracelular de lipídios no pré-adipócito.

Foi demonstrado que a fucoxantina tem um papel importante no controlo da obesidade, através da expressão da UCP1. Uma das principais limitações da presente pesquisa foi o facto da maioria dos estudos terem utilizado modelos animais com doses diferentes administradas. No entanto, existem alguns estudos realizados em ratos que demonstram que o $\mathrm{DL}_{50}$ é provavelmente superior a $2000 \mathrm{mg} / \mathrm{kg}$ de peso.

Considerando o objetivo previamente definido, foi possível caracterizar os processos de metabolização e toxicidade, bem como os mecanismos de ação da fucoxantina, enquanto composto anti-obesogénico.

\section{PALAVRAS-CHAVE}

Efeito anti-inflamatório, Fucoxantina, Obesidade, Tecido adiposo, UCP1

ABSTRACT

Fucoxanthin is one of the most abundant carotenoids in the marine environment, and it can be found in microalgae and macroalgae. Currently, there has been an increase in studies with this compound, due to its multiple health benefits, namely its anti-obesogenic effect. The present narrative review article pretends to caracterize the metabolization processes and toxicity, as well as the mechanisms of action of fucoxanthin in the control of obesity.

This research was conducted in the database "PubMed" using "fucoxanthin", "anti-inflammatory effect" and "obesity" as keywords. From the mentioned source, we analyzed 44 articles and selected 28, from 2015 to 2020.

After administration of fucoxanthin, it's rapidly metabolized to fucoxanthinol. Part of this metabolite is subsequently dehydrogenated to amarouciaxanthin A in the liver. Studies found that fucoxanthin has therapeutic properties, with a notable role in obesity. Additionally, it verified that fucoxanthin acts in this process, stimulating UCP1 expression, reducing white adipose tissue and inhibiting the intracellular accumulation of lipids in the pre-adipocyte.

It demonstrated that fucoxanthin could have a role in the control of obesity, through the expression of UCP1. One of the significant imitations of the present research was the fact that most of the studies used a non-human model and different doses. Nevertheless, there are some studies carried out on mice that demonstrate $L_{5}$ is probably higher than $2000 \mathrm{mg} / \mathrm{kg}$ of weight. According to the previously defined objective, it was possible to caracterize the metabolization processes and toxicity, as well as the mechanisms of action of fucoxanthin, as an anti-obesogenic compound.

KEYWORDS

Anti-inflammatory effect, Fucoxanthin, Obesity, Adipose tissue, UCP1 


\section{INTRODUÇÃO}

A fucoxantina é um dos carotenoides marinhos mais abundantes, representando mais de $10 \%$ da produção total estimada destes no ambiente marinho, podendo ser encontrada em microalgas tais como Phaeodactylum tricornutum, Cylindrotheca closterium, Isochrysis galbana e diatomáceas, e em macroalgas como na Undaria pinnatifida e Laminaria japonica (1-10). Trata-se de um pigmento acastanhado que confere essa cor característica às algas que o contêm, sendo obtido a partir dos cloroplastos das mesmas (1).

Devido à sua estrutura única, a fucoxantina apresenta diversas propriedades terapêuticas e farmacológicas, nomeadamente propriedades antiobesogénicas, antitumorais, antidiabéticas, antioxidantes, anti-inflamatórias e antiangiogénicas. Além disso, este composto tem efeitos protetores ao nível hepático, cardíaco e cerebral (6, 8, 11-14).

Tendo em consideração o conhecimento a priori da associação entre o carotenoide e o seu efeito anti-inflamatório, considerado como uma propriedade major, o objetivo da presente revisão narrativa é caracterizar os processos de metabolização e toxicidade, bem como os mecanismos de ação da fucoxantina no controlo da obesidade. Após a análise bibliográfica constatou-se a existência de múltiplas propriedades associadas a este carotenoide, no entanto a evidência do seu efeito antiobesogénico demonstrou ter uma maior relevância pelo facto de a obesidade ser considerada uma pandemia mundial.

\section{METODOLOGIA}

Esta revisão foi realizada na base de dados "PubMed" usando os descritores "fucoxantina" e "efeito anti-inflamatório" em simultâneo, e posteriormente foi utilizado o descritor "obesidade" para uma pesquisa mais direcionada. A partir da fonte mencionada, dos 476 artigos publicados entre 2015 e 2020, selecionaram-se 44 artigos a partir da leitura do título e do resumo, atendendo à inclusão das propriedades da fucoxantina nos mesmos. Após a leitura e análise integral dos artigos selecionados, utilizaram-se 28 artigos para a elaboração desta revisão, de acordo com o seu nível de pertinência e enquadramento com a temática a abordar.

\section{Metabolização}

Aquando da administração de fucoxantina, esta é rapidamente metabolizada por enzimas digestivas, especificamente pelas lípases e colesterol-esterases, que a transformam em fucoxantinol, por desacetilação, durante a digestão intestinal $(14,15)$. Parte deste metabolito é posteriormente desidrogenado/isomerizado para amarouciaxantina A no fígado. A amarouciaxantina A é armazenada no tecido adiposo branco, enquanto que o fucoxantinol entra na corrente sanguínea e podendo ser armazenado em vários órgãos (Figura 1) $(5-8,16,17)$.

A fucoxantina é instável em condições ácidas, pelo que alguns autores sugerem que este pigmento não seja totalmente utilizado pelo organismo, devido à digestão gástrica e intestinal (6). Além disso, como a fucoxantina é hidrofóbica, a sua absorção ocorre a nível intestinal através das mesmas vias utilizadas pelas gorduras alimentares $(12,18)$. Portanto, a taxa de absorção da fucoxantina pode ser influenciada pela composição da matriz alimentar, tendo-se verificado uma melhor solubilidade em triglicerídeos de cadeia média (MCT) ou no óleo de peixe (7).

Desta forma, diferentes estratégias têm vindo a ser desenvolvidas para aumentar a eficácia da absorção da fucoxantina nomeadamente associando-a a compostos lipídicos para melhorar o seu transporte através das barreiras celulares, aumentando a sua biodisponibilidade, ou pelo encapsulamento deste carotenoide em lipossomas ou micelas $(1,12)$.

\section{Figura 1}

Produtos da Metabolização da Fucoxantina (7)

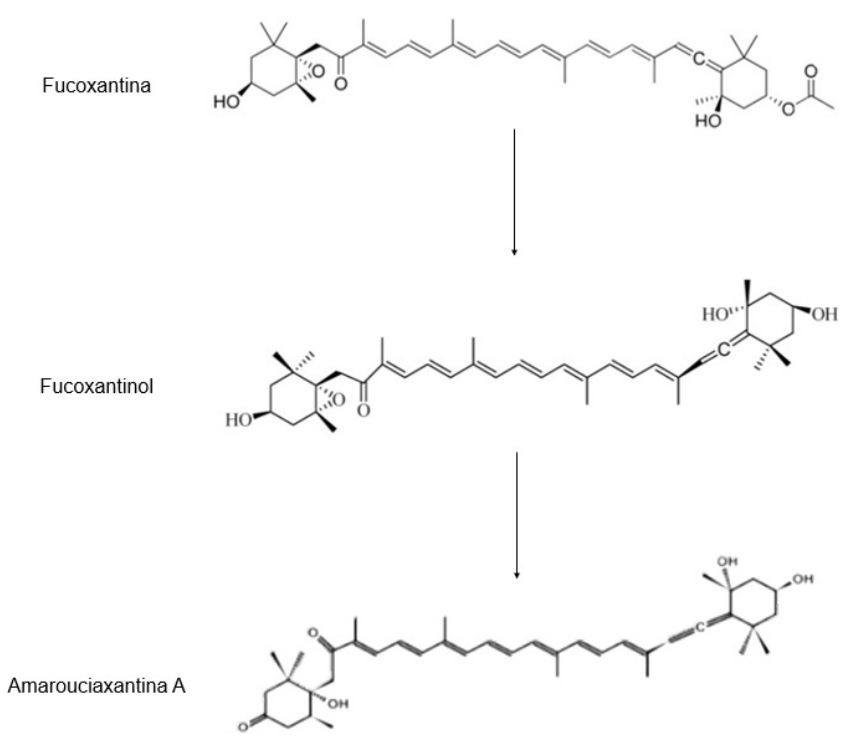

Num estudo realizado em ratos, verificou-se que uma dieta enriquecida em fucoxantina e óleo de peixe reduzia significativamente o ganho de peso, com consequente diminuição do tecido adiposo branco, quando comparado com ratos alimentados com uma dieta enriquecida apenas com fucoxantina (10). Paralelamente, foi demonstrado que um regime alimentar suplementado em fucoxantina e em ácido linoléico conjugado, diminui os níveis séricos de triglicerídeos, glicose e leptina em ratos obesos. Esta combinação obteve também os mesmos resultados em relação à perda de peso (10).

\section{Mecanismo de ação da fucoxantina}

A estrutura da fucoxantina confere-lhe uma variedade de atividades biológicas, já supracitadas anteriormente. Entre todas as propriedades terapêuticas e farmacológicas, o seu papel na obesidade é o mais notável $(9,19-21)$.

A Organização Mundial da Saúde (OMS) define obesidade e excesso de peso como uma acumulação anormal ou excessiva de gordura no tecido adiposo, o que representa um risco para a saúde. Esta comorbilidade induz um estado de inflamação, que é determinante no desenvolvimento de complicações como resistência à insulina, diabetes tipo 2, arteriosclerose e esteatose hepática não alcoólica (10). Neste seguimento, várias estratégias têm vindo a ser desenvolvidas na tentativa de combater este grave problema de saúde pública, no qual a fucoxantina pode ter uma especial relevância $(10,22)$.

Os adipócitos são células endócrinas que secretam mediadores biologicamente ativos, tais como as adipocinas, que incluem a leptina, adiponectina, resistina, fator de necrose tumoral-a (TNF-a) e a proteína quimioatrativa de monócitos-1 (MCP-1) $(10,23)$. O tecido adiposo apresenta dois tipos de adipócitos, os brancos e os castanhos (3). O tecido adiposo branco armazena as calorias em excesso como gordura, levando à obesidade (17). Pelo contrário, os adipócitos castanhos mantêm a temperatura corporal, utilizando as calorias em excesso, armazenadas sob a forma de gordura, para produzir calor. Esta atividade termogénica é realizada pela "proteína desacopladora 1" (UCP1), presente nas membranas mitocondriais internas do tecido adiposo castanho, sendo, assim, responsável pela diminuição da gordura acumulada no tecido adiposo branco $(3,7,22)$.

Estudos em humanos mostram que a fucoxantina atua neste processo, estimulando a expressão da UCP1, reduzindo o tecido adiposo branco 
e inibindo a acumulação intracelular dos lípidos no pré-adipócito $(24,25)$. Adicionalmente, bloqueia a diferenciação dos adipócitos, e suprime a expressão de várias citocinas pró-inflamatórias, tais como TNF-a e a MCP-1, o que conduz à diminuição da resistência à insulina e à redução da glicémia $(3,12)$.

Considerando que a leptina encontra-se em concentrações proporcionais à dimensão e ao número de adipócitos. Em indivíduos obesos, as concentrações de leptina estão aumentadas comparativamente aos indivíduos normoponderais, no entanto esta hormona é incapaz de desempenhar as suas funções, nomeadamente a manutenção da homeostasia do tecido adiposo e o consequente controlo do consumo alimentar e do gasto energético. Esta resistência à leptina, inerente ao excesso de peso, ocorre devido ao não reconhecimento pelos seus recetores. Um estudo realizado em ratos sugere que a fucoxantina diminui a expressão desta hormona, o que se traduz na amplificação dos seus efeitos antiobesogénicos (12).

Para além do mecanismo anteriormente mencionado, a fucoxantina e o seu metabolito, fucoxantinol, inibem a absorção dos lípidos, interferindo no seu metabolismo, através da inativação da lípase pancreática. De notar que, a atuação da fucoxantina e do fucoxantinol, em ratos, foram semelhantes. No entanto, estes resultados foram menos significativos, comparativamente aos que são atingidos quando administrado o principal fármaco usado no tratamento da obesidade (10).

A fucoxantina, relativamente ao perfil lipídico, reduz significativamente as concentrações plasmáticas e hepáticas de triglicerídeos e influencia de forma positiva as enzimas reguladoras do colesterol (25). Este carotenoide potencia a $\beta$-oxidação dos ácidos gordos nas mitocôndrias musculares e cardíacas, impedindo o aumento dos ácidos gordos livres e consequentemente a acumulação de gordura no músculo-esquelético, caraterístico da obesidade e de outras doenças metabólicas $(10,12,13)$. Tal processo ocorre devido à regulação positiva da carnitina aciltransferase 1 (CPT1), enzima mitocondrial responsável pela formação de acilcarnitinas, a qual catalisa a reação que transfere o grupo acil da acil-CoA para a $L$-carnitina, resultando numa etapa essencial da $\beta$-oxidação dos ácidos gordos $(7,10,12)$.

\section{Toxicidade}

Tendo em vista o uso potencial da fucoxantina como nutracêutico, especialmente pela sua atividade antiobesogénica, vários estudos de toxicidade foram conduzidos a fim de avaliar a sua segurança (10, 12, 26). No entanto, os estudos em humanos são limitados. Existem apenas alguns estudos realizados em ratos que demonstraram que a $\mathrm{DL}_{50}$ é provavelmente superior a $2000 \mathrm{mg} / \mathrm{kg}$ de peso (27).

Loy et al. verificaram que ratos alimentados com doses orais repetidas de fucoxantina com 95\% de pureza, durante quatro semanas, não demostraram toxicidade significativa. Além disso, constataram a não ocorrência de mortalidade nem de anormalidades, aquando da realização de testes de toxicidade, com doses únicas de fucoxantina, compreendidas entre 1000 a $2000 \mathrm{mg} / \mathrm{kg}$ de peso, bem como em doses repetidas de 500 a $1000 \mathrm{mg} / \mathrm{kg}$ de peso, durante 30 dias. Contudo, foi observado secundariamente um aumento significativo no colesterol total plasmático, nos ratos alimentados com fucoxantina (10).

\section{ANÁLISE CRÍTICA}

As algas fazem parte do padrão alimentar da cultura asiática, particularmente no Japão e Coreia (28). No entanto, devido à globalização, o seu consumo tem vindo a aumentar a nível mundial, nomeadamente através do consumo de sushi. As principais algas marinhas consumidas são o Wakame (Undaria pinnatifida) e o
Kombu (Laminaria japónica). Porém, as microalgas marinhas, como Phaeodactylum tricornutum e Isochrysis galbana, têm maior conteúdo de fucoxantina, tendo sido consideradas como potenciais produtores comerciais deste composto (10).

Com esta revisão narrativa, em sentido lato, comprovou-se a existência de uma relação positiva entre a fucoxantina e o seu efeito no controlo da obesidade, quer em humanos quer em ratos, através da expressão da UCP1, o que conduz a uma redução do tecido adiposo branco e supressão da expressão de várias citocinas pró-inflamatórias.

A fucoxantina tem, também, um papel importante na inibição da lípase pancreática, constatando-se que a sua ação é semelhante à de um dos fármacos mais utilizados no tratamento da obesidade, embora com efeitos menos significativos. Assim, este carotenoide pode ser possivelmente utilizado como uma terapia coadjuvante a este tipo de fármacos.

Todavia, verificaram-se limitações na presente pesquisa, sobretudo o facto da maioria dos estudos terem utilizado modelos não humanos e das doses administradas de fucoxantina serem diferentes. Adicionalmente a biodisponibilidade deste carotenoide mostrou ser baixa em seres humanos, daí a necessidade de explorar novos sistemas de administração da fucoxantina, nomeadamente o seu encapsulamento em lipossomas ou micelas. No que concerne à toxicidade, também constituiu uma limitação, devido à escassez de estudos experimentais que testam a segurança das doses administradas.

Relativamente aos suplementos alimentares, já existe uma gama de produtos que contêm este pigmento. Estes suplementos demonstraram provocar uma redução da gordura corporal e hepática (10). Adicionalmente, suprimem a diferenciação de adipócitos e a acumulação de lípidos, através de uma regulação negativa na adipogénese (10).

\section{CONCLUSÕES}

Atendendo ao objetivo previamente definido, foi possível caracterizar o processo metabólico da fucoxantina, bem como os seus mecanismos de ação, que desempenham um papel importante no controlo da obesidade.

As algas marinhas, ricas em fucoxantina, podem ser integradas na dieta, dado o seu contributo benéfico para a manutenção de um peso corporal saudável, e consequente possível redução da incidência da obesidade, considerando os resultados positivos encontrados nos escassos estudos realizados em humanos.

\section{REFERÊNCIAS BIBLIOGRÁFICAS}

1. Viera I, Gálvez A, Roca M. Bioaccessibility of marine carotenoids. Mar Drugs. 2018;16(10):1-21.

2. Barbalace MC, Malaguti M, Giusti L, Lucacchini A, Hrelia S, Angeloni C. Antiinflammatory activities of marine algae in neurodegenerative diseases. Int $\mathrm{J}$ Mol Sci [Internet]. 2019 Jun 22 [cited 2020 Apr 23];20(12):3061. Available from: https://www. mdpi.com/1422-0067/20/12/3061.

3. Maeda H, Kanno S, Kodate M, Hosokawa M, Miyashita K. Fucoxanthinol, metabolite of fucoxanthin, improves obesity-induced inflammation in adipocyte cells. Mar Drugs. 2015;13(8):4799-813.

4. Wang J, Ma Y, Yang J, Jin L, Gao Z, Xue L, et al. Fucoxanthin inhibits tumourrelated lymphangiogenesis and growth of breast cancer. J Cell Mol Med. 2019 Mar 1;23(3):2219-29.

5. Zhang H, Tang Y, Zhang Y, Zhang S, Qu J, Wang X, et al. Fucoxanthin: a promising medicinal and nutritional ingredient. http://dx.doi.org/101155/2015/431047. 2015;2015:2-4.

6. Guo B, Oliviero T, Fogliano V, Ma Y, Chen F, Capuano E. Gastrointestinal Bioaccessibility and Colonic Fermentation of Fucoxanthin from the Extract of the Microalga Nitzschia laevis. J Agric Food Chem. 2019;68(7):1844-50. 
7. Gammone M, D'Orazio N. Anti-obesity activity of the marine carotenoid fucoxanthin. Mar Drugs [Internet]. 2015 Apr 13 [cited 2020 Apr 23];13(4):2196-214. Available from: http://www.mdpi.com/1660-3397/13/4/2196

8. Zhang Y, Wu H, Wen H, Fang H, Hong Z, Yi R, et al. Simultaneous determination of fucoxanthin and its deacetylated metabolite fucoxanthinol in rat plasma by liquid chromatography-tandem mass spectrometry. Mar Drugs. 2015 Oct;13(10):6521-36. 9. Guo B, Liu B, Yang B, Sun P, Lu X, Liu J, et al. Screening of diatom strains and characterization of Cyclotella cryptica as a potential fucoxanthin producer. Mar Drugs [Internet]. 2016 Jul 8 [cited 2020 Apr 23];14(7):125. Available from: http://www.mdpi. com/1660-3397/14/7/125.

10. Loy C, Moi P. Marine algae as a potential source for anti-obesity agents. Mar Drugs [Internet]. 2016 Dec 7 [cited 2020 Apr 23];14(12):222. Available from: http://www.mdpi. com/1660-3397/14/12/222.

11. Chen J, Lee J, Lin B, Peng Y, Liu J, Chen S, et al. Protective effects of fucoxanthin on ultraviolet B-induced corneal denervation and inflammatory pain in a rat model. Mar Drugs. 2019 Mar 5;17(3).

12. Koo S, Hwang J, Yang S, Um J, Hong K, Kang K, et al. Anti-obesity effect of standardized extract of microalga phaeodactylum tricornutum containing fucoxanthin. Mar Drugs. 2019 May; 17(5).

13. Talero E, García-Mauriño S, Ávila-Román J, Rodríguez-Luna A, Alcaide A, Motilva V. Bioactive compounds isolated from microalgae in chronic inflammation and cancer. Vol. 13, Marine Drugs. MDPI AG; 2015. p. 6152-209.

14. Chen J, Lee J, Lin B, Liu J, Huang Y, Chen Z, et al. Inhibition of ultraviolet B-induced expression of the proinflammatory cytokines TNF-a and VEGF in the cornea by fucoxanthin treatment in a rat model. Mar Drugs. 2016 Jan 1;14(1).

15. Taira J, Sonamoto M, Uehara M. Dual biological functions of a cytoprotective effect and apoptosis induction by bioavailable marine carotenoid fucoxanthinol through modulation of the nrf2 activation in raw264.7 macrophage cells. Mar Drugs. 2017 Oct;15(10).

16. Lin H, Tsou Y, Chen Y, Lu W, Hwang P. Effects of low-molecular-weight fucoidan and high stability fucoxanthin on glucose homeostasis, lipid metabolism, and liver function in a mouse model of type II diabetes. Mar Drugs. 2017 Apr;15(4).

17. Gille A, Stojnic B, Derwenskus F, Trautmann A, Staiger U, Posten C, et al. A lipophilic fucoxanthin-rich phaeodactylum tricornutum extract ameliorates effects of diet-induced obesity in C57BL/6J mice. Nutrients. 2019 Apr;11(4):1-17.

18. Martin L. Fucoxanthin and its metabolite fucoxanthinol in cancer prevention and treatment. Mar Drugs. 2015 Aug 1;13(8):4784-98.

19. Zhang L, Wang H, Fan Y, Gao Y, Li X, Hu Z, et al. Fucoxanthin provides neuroprotection in models of traumatic brain injury via the Nrf2-ARE and Nrf2-autophagy pathways. Sci Rep. 2017;7.

20. Chang M, Li L, Lin C. Fucoidan-fucoxanthin ameliorated cardiac function via IrS1/ Grb2/ SOS1, GSK3ß/CREB pathways and metabolic pathways in senescent mice. Mar Drugs. 2019 Jan 21;17(1):18.

21. Chau T, Chen $Y$, Lin H, Hsia M. Preventive effects of fucoidan and fucoxanthin on hyperuricemic rats induced by potassium oxonate. Mar Drugs. 2019;17(6):1-14. 22. Mounien L, Tourniaire F, Landrier J. Anti-obesity effect of carotenoids: direct impact on adipose tissue and adipose tissue-driven indirect effects. Nutrients. 2019 Jul;11(7):1562.

23. Hwang A, Phan N, Lu J, Hieu N, Lin C. Low-molecular-weight fucoidan and high-stability fucoxanthin from brown seaweed exert prebiotics and anti-inflammatory activities in Caco-2 cells. Food Nutr Res. 2016;60(February).

24. Stonik V, Stonik I. Low-molecular-weight metabolites from diatoms: structures, biological roles and biosynthesis. Vol. 13, Marine Drugs. MDPI AG; 2015. p. 3672-709. 25. Maeda H, Fukuda S, Izumi H, Saga N. Anti-oxidant and fucoxanthin contents of brown alga Ishimozuku (Sphaerotrichia divaricata) from the West Coast of Aomori, Japan. Mar Drugs. 2018 Jul;16(8):255.

26. Martínez K, Lauritano C, Romano G, lanora A. Marine microalgae with anti-cancer properties. Mar Drugs. 2018 May;16(5):165.

27. Beppu F, Niwano Y, Tsukui T, Hosokawa M, Miyashita K. Single and repeated oral dose toxicity study of fucoxanthin (FX), a marine carotenoid, in mice. J Toxicol Sci.
2009 Oct;34(5):501-10

28. Terasaki M, Masaka S, Fukada C, Houzaki M, Endo T, Tanaka T, et al. Salivary glycine is a significant predictor for the attenuation of polyp and tumor microenvironment formation by fucoxanthin in AOM/DSS mice. In Vivo (Brooklyn). 2019;33(2):365-74. 